\title{
Reaching the EeV frontier in neutrino-nucleon cross sections in upcoming neutrino telescopes
}

\author{
Victor B. Valera ${ }^{a, *}$ and Mauricio Bustamante ${ }^{a}$ \\ ${ }^{a}$ Niels Bohr International Academy, Niels Bohr Institute, University of Copenhagen, \\ DK-2100 Copenhagen, Denmark \\ E-mail: vvalera@nbi.ku.dk, mbustamante@nbi.ku.dk
}

Measuring neutrino interactions with matter is arduous but rewarding. To date, experiments have measured the neutrino-nucleon cross section in the MeV-PeV range, using terrestrial and astrophysical neutrinos. We endeavor to push that measurement to the EeV scale, in order to test competing expectations of the deep structure of nucleons and possibly reveal new neutrino interactions. Cosmogenic neutrinos, long-sought but still undiscovered, provide the only feasible way forward. However, because their flux is low, they have evaded detection so far. Fortunately, upcoming in-ice radio-detection neutrino telescopes, like RNO-G and the radio component of IceCube-Gen2, have a real chance of discovering them in the next 10-20 years. In preparation, we perform the first detailed study of their sensitivity to the deep-inelastic-scattering neutrino-nucleon cross section at EeV energies, extracted from the attenuation of the cosmogenic neutrino flux as it traverses the Earth across different directions. We use up-to-date predictions and tools at every step: in the flux of cosmogenic neutrinos-predicted using recent ultra-high-energy cosmicray measurements - in their propagation inside the Earth - computed using leading and subleading neutrino interactions - and in their detection in radio-based neutrino telescopes - based on advanced simulated detector responses. We report the preliminary sensitivity of IceCube-Gen2.

$37^{\text {th }}$ International Cosmic Ray Conference (ICRC 2021)

July 12 th - 23rd, 2021

Online - Berlin, Germany

\footnotetext{
${ }^{*}$ Presenter
} 


\section{Introduction}

Neutrinos have always been harbingers of new insight into fundamental physics. Neutrino interactions with matter, though feeble, are of great importance for particle and astroparticle physics. We make the first detailed forecast of our near-future capability to measure the neutrino-nucleon cross section $\left(\sigma_{v N}\right)$ in the $\mathrm{EeV}$ energy regime, for the first time, with the next generation of neutrino telescopes, in particular, with the largest planned in-ice neutrino radio telescope, IceCube-Gen2 [1].

Ultra-high-energy neutrinos (UHEv), with EeV-scale energies, have been long predicted, but have not yet been discovered, on account of their low predicted flux. The cosmogenic neutrinos produced in the scattering of ultra-high-energy cosmic rays (UHECRs) on the cosmic microwave background (CMB) are a low, but guaranteed flux of UHE neutrinos that could help reveal the origin of cosmic rays [2]. In addition, there might a higher flux of UHE astrophysical neutrinos, produced directly inside UHECR sources (galaxy clusters, pulsars, AGNs, GRBs, etc.). Beyond their role as tracers of UHECRs sources, UHE $v$ may probe the Standard Model (SM) at energies otherwise unreachable, including testing its many proposed high-energy extensions.

Accelerator experiments measured the neutrino-nucleon cross section up to $E_{v} \sim 350 \mathrm{GeV}$ [3], while future accelerator experiments aim to reach $E_{v} \sim 10^{3} \mathrm{GeV}$. Using IceCube data, measurements reached the TeV-PeV scale for the first time [4-6], where the main contribution to the cross section comes from the deep inelastic scattering of neutrinos off the partons in the nucleon. As neutrinos above $\mathrm{TeV}$ energies propagate through the Earth they interact with matter, losing energy or being absorbed. This attenuates the neutrino flux that reaches the detector, depending on the neutrino energy and arrival direction. From this, we extract the neutrino-nucleon cross section.

Now we aim to reach the next energy frontier by performing the first detailed study of the measurement capabilities of the next generation of neutrino detectors up to $E_{v} \sim 10^{10} \mathrm{GeV}$. (Ref. [7] forecast the sensitivity to the UHE $v_{\tau}$ cross section in GRAND and POEMMA, in a simplified calculation framework.) Current theoretical estimations lose precision around $10^{8} \mathrm{GeV}$ due to the limited of knowledge of the parton distribution functions (PDFs). UHE $v$ would allow for the first measurement of the neutrino-nucleon cross section in this energy regime, improving our understanding of the nucleon PDFs. Additionally, beyond-the-Standard-Model (BSM) physics might manifest as deviations from the cross section predicted by the SM. UHE $v$ are our only tool to potentially probe such models and refine our understanding of the SM at ultra-high energies.

We make the first detailed estimates of the sensitivity of the planned radio component of IceCube-Gen2 to measuring the UHE neutrino-nucleon cross section. We use state-of-the art ingredients at all stages: in the flux predictions, computation of the attenuation, and estimates of the detector response, including energy and angular uncertainties.

\section{The ultra-high-energy neutrino flux at Earth}

We consider three benchmark fluxes of UHEv, with energies in the EeV scale: cosmogenic neutrinos and astrophysical neutrinos-for which we use the most up-to-date flux predictions-and the IceCube 8-year $v_{\mu}$ power-law flux [8], extrapolated to high energies.

Cosmogenic, or GZK, neutrinos [2] are produced in the interaction of UHECRs, en route to Earth, with the cosmological microwave background (CMB) and the extragalactic background 


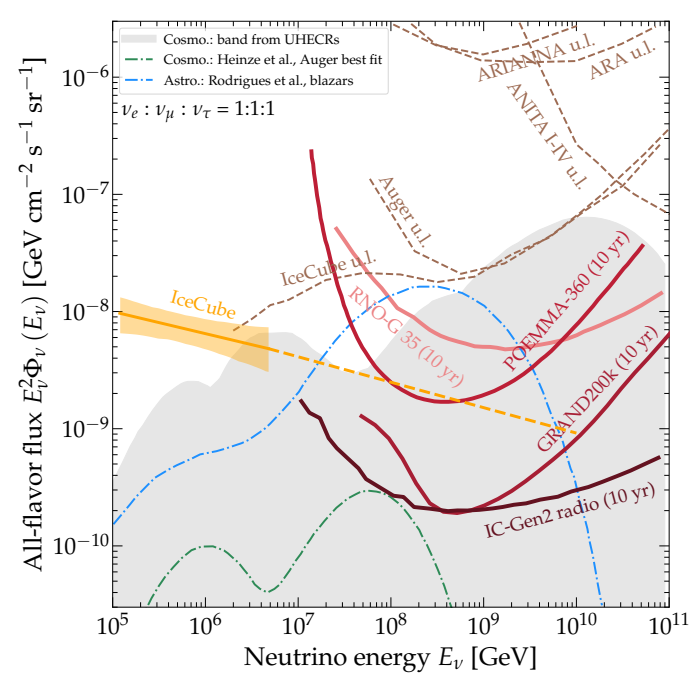

Figure 1: Cosmogenic ("cosmo.") [9, 11, 12] and astrophysical source ("astro.") [10, 13] UHE $v$ fluxes. For reference, we include the IceCube 8-year measurement of the high-energy astrophysical neutrino flux with through-going muons [8], and upper limits and sensitivity estimates, respectively, of current and future detectors.

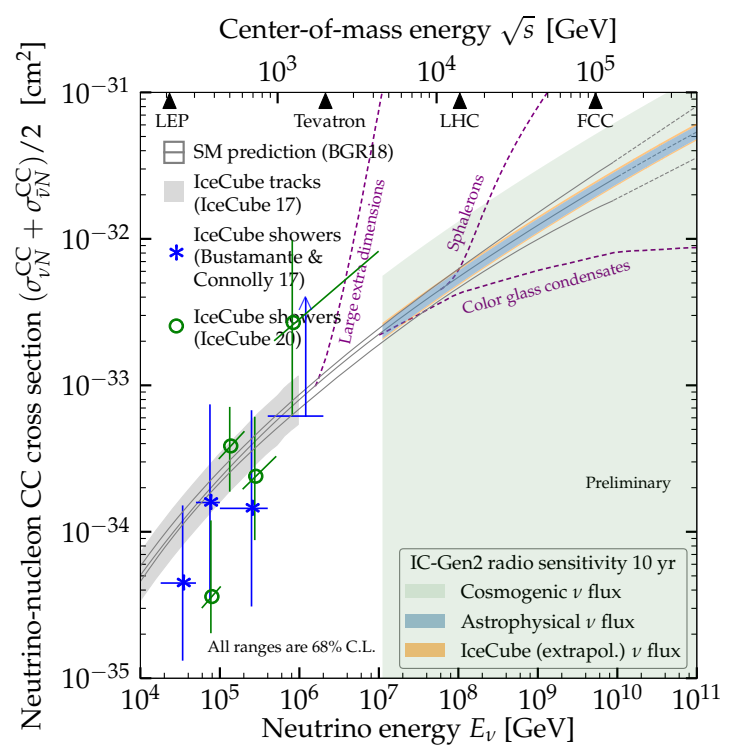

Figure 2: Neutrino nucleon cross section $\left(\sigma_{v N}\right)$ inferred from IceCube tracks [4, 5] and showers [5] events. We show the state-of-the-art Standard Model prediction from [14]. Figure adapted from Ref. [15].

light (EBL). The photopion interaction of an $\sim \mathrm{EeV}$ proton with a low-energy $\mathrm{CMB}$ or EBL photon produces $v_{e}$ and $v_{\mu}$ with energies in the $\mathrm{EeV}$ regime. As a benchmark, we use the cosmogenic flux prediction from Ref. [9], based on fits to UHECR observations in Auger.

Astrophysical UHEv are produced in the interaction of UHECRs with ambient matter inside their sources. Their flux is higher due to additional production processes, like multi-pion production. As a benchmark, we use the maximum astrophysical flux prediction from AGN from Ref. [10].

Figure 1 shows our three benchmark fluxes. We assume flavor equipartition by the time the fluxes reach the Earth and equal number of neutrinos of anti-neutrinos of each flavor. We extract the cross section assuming each of the three benchmark UHE $v$ fluxes in turn.

\section{Ultra-high-energy neutrino propagation inside the Earth}

Figure 2 shows the high-energy neutrino-nucleon cross section measurements up to PeV energies, which is consistent with the SM prediction. The Standard Model predicts a large neutrinonucleon cross section at EeV neutrino energies [14, 16-19]. When forecasting the sensitivity to cross-section measurements, we adopt the state-of-the-art BGR18 deep-inelastic-scattering (DIS) neutrino-nucleon cross-section $\left(\sigma_{v N}\right)$ from Ref. [14] as our reference value; we also explore the sensitivity to larger and smaller cross sections. Because the cross section grows with energy, a flux of UHE $v$ is attenuated as it propagates inside the Earth. Broadly stated, the higher $\sigma_{v N}$, the stronger the in-Earth attenuation. We propagate the UHEv flux from the surface of the Earth, through its interior, where we model its matter density using the Preliminary Reference Earth Model, and up to 

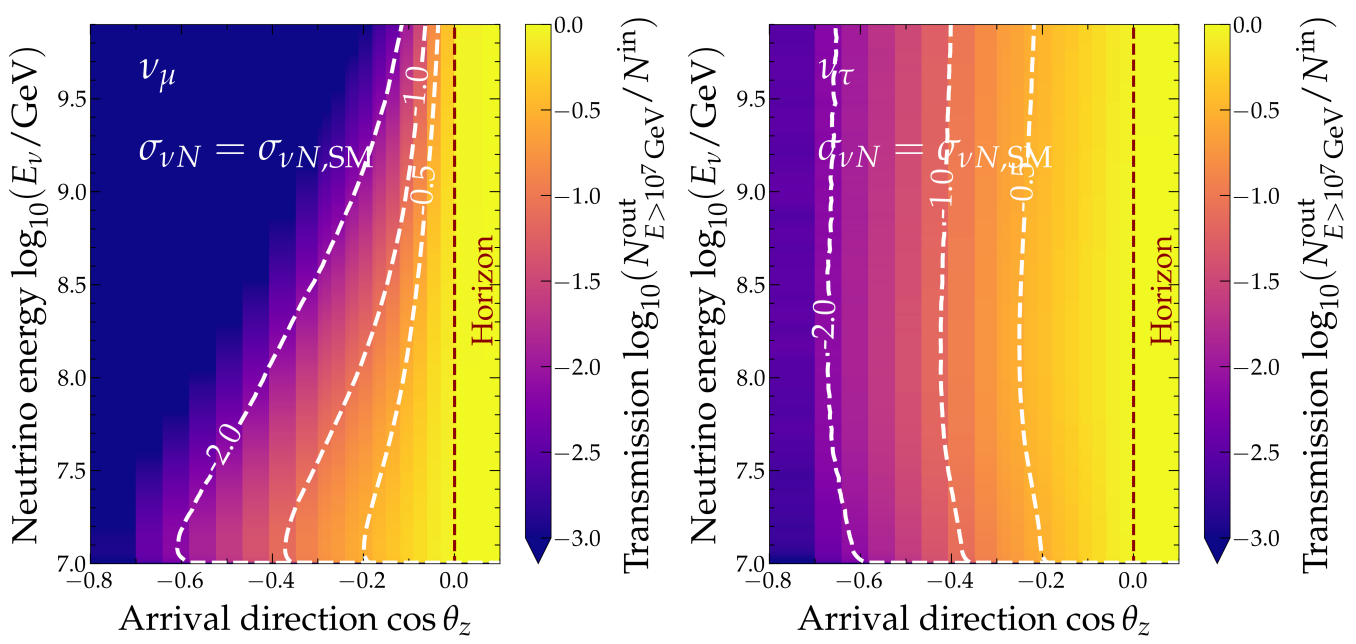

Figure 3: High-energy neutrino transmission probability, i.e., the number of surviving neutrinos for different energies and different arrival directions to the detector, as a function of the zenith angle $\left(\theta_{z}\right)$. We show the transmission of neutrinos above $10 \mathrm{PeV}$ for a $v_{\mu}$ (left) and $v_{\tau}$ (right) beam after propagation inside the Earth.

IceCube-Gen2, to obtain the neutrino spectrum that reaches the detector, from all possible incoming directions.

To propagate the neutrino flux we use NuPropEARTh [20], which uses the state-of-the-art computations of the neutrino-matter cross sections [14], including the leading contribution from $v N$ DIS, and the contributions from sub-leading interactions like scattering off the photon field of nucleons and nuclei, and the scattering off atomic electrons. The in-Earth attenuation depends on energy, direction, and flavor, and so does the flux that reaches the detector, i.e., $\Phi_{v_{\alpha}}^{\operatorname{det}}\left(E_{v}, \cos \theta_{z}\right)$. At UHE, differences between the attenuation of neutrinos and anti-neutrinos are tiny; even so, we propagate neutrinos and anti-neutrinos of each flavor separately.

Figure 3 shows the fraction of surviving neutrinos with energies above $10 \mathrm{PeV}$, after propagating inside the Earth, based on simulations of injected mono-energetic neutrino beams that reach IceCube-Gen2 from different directions. We express the beam direction via the zenith angle $\theta_{z}$ : $\cos \theta_{z}<1$ for upgoing neutrinos, which traverse up to the diameter of the Earth; $\cos \theta_{z}>1$ for downgoing neutrinos, which traverse only the layer of ice between the surface of the Earth and the underground location of IceCube-Gen2; and $\cos \theta_{z}=0$ for neutrinos coming from the horizontal direction, which still traverse many kilometers inside the Earth before reaching IceCube-Gen2. During propagation, the originally mono-energetic beam spreads out into a spectrum of lower energies. The right panel of Fig. 3 shows the effect of tau regeneration, that is, the production and subsequent decay of high-energy taus inside the Earth, leading to the production of $v_{\tau}$ at lower energies.

Figure 4 shows the attenuation for different choices of the $v N$ DIS cross section. We only modify the DIS contribution to the total cross section, leaving sub-leading contributions unmodified. To estimate the sensitivity of IceCube-Gen2, we propagate neutrinos inside the Earth assuming that $\sigma_{v N}$ is enhanced with respect to the central value of the BGR18 SM prediction, $\sigma_{v N}$,SM, by a factor $f$ that is independent of the neutrino energy. 

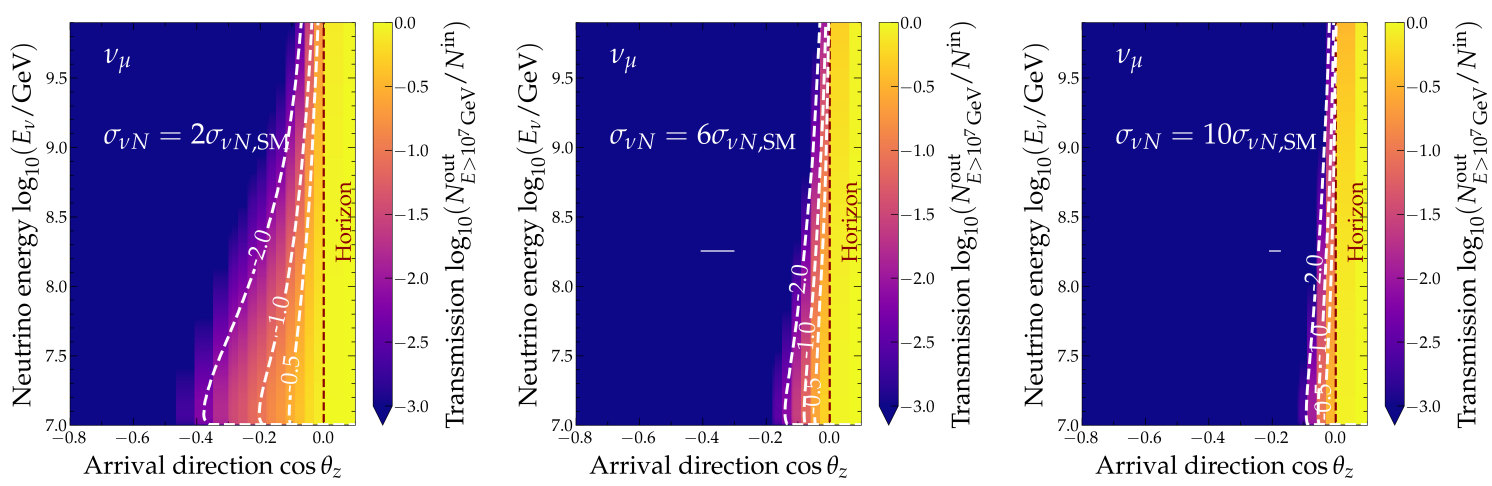

Figure 4: Neutrino transmission probability for different scenarios of enhancement of the Standard Model deep inelastic neutrino-nucleon cross section.

\section{Event rate estimation in the radio component of IceCube-Gen2}

The radio component of IceCube-Gen2 is a planned extension of IceCube [1]. It consists of an array of underground radio detectors covering $\sim 500 \mathrm{~km}^{2}$. It will detect the coherent radio emission from showers triggered by UHE $v$.

The number of detected neutrinos coming from a given direction in IceCube-Gen 2 is $\propto$ $\Phi_{v_{\alpha}}^{\text {Earth }}\left(E_{v}\right) \sigma_{v N}\left(E_{v}\right) \exp \left(-\tau_{v N}\left(E_{v}, \cos \theta_{z}\right)\right)$. Here, $\tau_{v N}$ is the Earth opacity to neutrino-matter interactions and is proportional to $\sigma_{v N}$ and to the Earth column density traversed by the neutrino. Therefore, our sensitivity to $\sigma_{v N}$ stems from the interplay of two competing effects: a larger cross section implies a stronger flux attenuation, but also increases linearly the probability of neutrino detection. The contribution from downgoing neutrinos $\left(\cos \theta_{z}>0\right)$ is dominant since they reach the detector mostly unattenuated ( $\left.e^{\tau_{\nu N}} \sim 1\right)$ making the event rate nearly linear in $\sigma_{v N}$.

After an exposure time $T$, the differential rate of neutrino-induced events at IceCube-Gen2 is

$$
\frac{d^{2} N_{v_{\alpha}}^{\mathrm{CC}}}{d E_{v} d \cos \theta_{z}}=2 \pi T N_{\mathrm{Av}} \rho_{\mathrm{ice}} V_{\mathrm{eff}, v_{\alpha}}^{\mathrm{CC}}\left(E_{\nu}\right) \sigma_{v N}^{\mathrm{CC}}\left(E_{\nu}\right) \phi_{v_{\alpha}}^{\mathrm{det}}\left(E_{\nu}, \cos \theta_{z}\right)
$$

where $N_{\mathrm{Av}}$ is the Avogadro number, $\rho_{\text {ice }}$ is the ice density, and $V_{\mathrm{eff}, v_{\alpha}}^{\mathrm{CC}}$ is the direction-averaged effective volume of the radio component of IceCube-Gen2, i.e., its sensitivity to neutrinos, assuming it consists of 200 phased-array radio-detection stations, and scaled up from the RNO-G effective volume [21].

We rewrite the differential rate in terms of observable quantities: deposited shower energy $\left(E_{\mathrm{dep}}\right)$, and reconstructed shower direction $\left(\theta_{z, \text { rec }}\right)$. For that purpose, we multiply Eq. (1) by the Gaussian resolution functions $R_{E_{\text {true }}}, R_{E_{\text {dep }}}$, and $R_{\theta_{z}}$. They represent, respectively, the connection between the true shower energy $\left(E_{\text {true }}\right)$ and neutrino energy $\left(E_{v}\right)$, between the deposited energy $\left(E_{\mathrm{dep}}\right)$ and the true energy $\left(E_{\text {true }}\right)$, and between the reconstructed arrival direction $\left(\cos \theta_{z, \text { rec }}\right)$ and the real arrival direction $\left(\cos \theta_{z}\right)$. For $R_{E_{\text {true }}}$, we use the energy-dependent width on $\log _{10}\left(E_{\text {true }} / E_{v}\right)$ from Ref. [22], which accounts for the inelasticity distribution of $v N$ DIS and for detector effects; it varies from $\sim 22 \%$ at $10^{8} \mathrm{GeV}$ to $\sim 40 \%$ at $10^{10} \mathrm{GeV}$. For $R_{E_{\text {dep }}}$, we use the energy-dependent width from Ref. [22], which varies between $8 \%$ at $10^{8} \mathrm{GeV}$ to $15 \%$ at $10^{9} \mathrm{GeV}$. For $R_{\theta_{z}}$, we adopt 
a benchmark width of $5^{\circ}$ in $\theta_{z}$. Thus, we write the event rate in terms of observable quantities as

$$
\begin{array}{r}
\frac{d^{2} N_{v_{\alpha}}^{\mathrm{CC}}}{d E_{\mathrm{dep}} d \cos \theta_{z, \text { rec }}}=\int_{-1}^{+1} d \cos \theta_{z} \int d E_{v} \int d E_{\text {true }} \frac{d^{2} N_{v_{\alpha}}^{\mathrm{CC}}}{d E_{v} d \cos \theta_{z}} R_{E_{\text {true }}}\left(E_{\text {true }}, E_{v}\right) \\
R_{E_{\text {dep }}}\left(E_{\text {dep }}, E_{\text {true }}, E_{v}\right) R_{\theta_{z}}\left(\cos \theta_{z, \text { rec }}, \cos \theta_{z}\right) .
\end{array}
$$

To obtain the total, full-sky event rate, we integrate Eq. (2) over $-1<\cos \theta_{z \text {,rec }}<1$, and over $E_{\mathrm{dep}}>1 \mathrm{PeV}$ for each of the six neutrino species $\left(v_{e}, \overline{v_{e}}, v_{\mu}, \overline{v_{\mu}}, v_{\tau}, \overline{v_{\tau}}\right)$, and add them all together.

Figure 5 shows the number of predicted events $N(f)$ as a function of the cross section enhancement factor $f$ for our three benchmark UHEv fluxes. For comparison, we draw the expected event rate assuming a linear growth, i.e., without in-Earth attenuation.

\section{Statistical methods}

Assuming in turn each of our three UHE $v$ fluxes from Fig. 1, we estimate the sensitivity to $\sigma_{v N}$ by answering the question of how precisely the value $f$ will be measured in IceCube-Gen2 if $N_{\text {obs }}$ events are detected in 10 years. We perform a Bayesian analysis using an unbinned Poissonian likelihood, i.e.,

$$
\mathcal{L}\left(f, N_{\text {obs }}\right)=\frac{e^{-\left[N(f)+N_{\text {bkg }}\right]}\left[N(f)+N_{\text {bkg }}\right]^{N_{\text {obs }}}}{N_{\text {obs }} !},
$$

where $N(f)$ is the number of expected events for an enhancement factor $f$, Eq. (2). The estimated background $N_{\mathrm{bkg}}$ is generated by atmospheric muons and is set to 0.311 per year [21]. We vary $N_{\text {obs }}$ between 1 and 1000, in integer steps. For each value of $N_{\text {obs }}$, we maximize the posterior $\mathcal{P}\left(f, N_{\text {obs }}\right)=\mathcal{L}\left(f, N_{\text {obs }}\right) \pi(f)$ with respect to $f$, using a flat prior $\pi(f)$ on $f$, and find the credible intervals at $68 \%, 95 \%$, and $99 \%$.

\section{Results}

Figure 6 shows our resulting estimated sensitivity of IceCube-Gen2 to the neutrino-nucleon cross section as a function of the number of future detected events assuming each of the benchmark flux models. For the cosmogenic scenario [9] the number of expected events is low and therefore the credible intervals are broad. For the astrophysical source and IceCube flux, the number of expected events is higher and the bounds are stronger. For example, assuming that the true cross section is the Standard Model cross section, we would be able to measure $\sigma_{v N} / \sigma_{v N}, \mathrm{SM}=1.0_{-1.0}^{+1.4}$ using cosmogenic neutrinos, $1.0_{-0.13}^{+0.15}$ using extrapolated IceCube flux, and $1.0_{-0.11}^{+0.10}$ using astrophysical neutrinos, after 10 years of IceCube-Gen2.

Figure 2 shows the predicted precision with which the cross section will be measured, compared to theoretical uncertainties. Our results show that if the UHEv flux is as low as the cosmogenic flux from Ref. [9], a measurement of the cross section will not be possible, but if it is as high as the astrophysical flux from Ref. [10], it could be measured to within $\sim 11 \%$, better than the present uncertainties in the cross-section predictions [14]. 


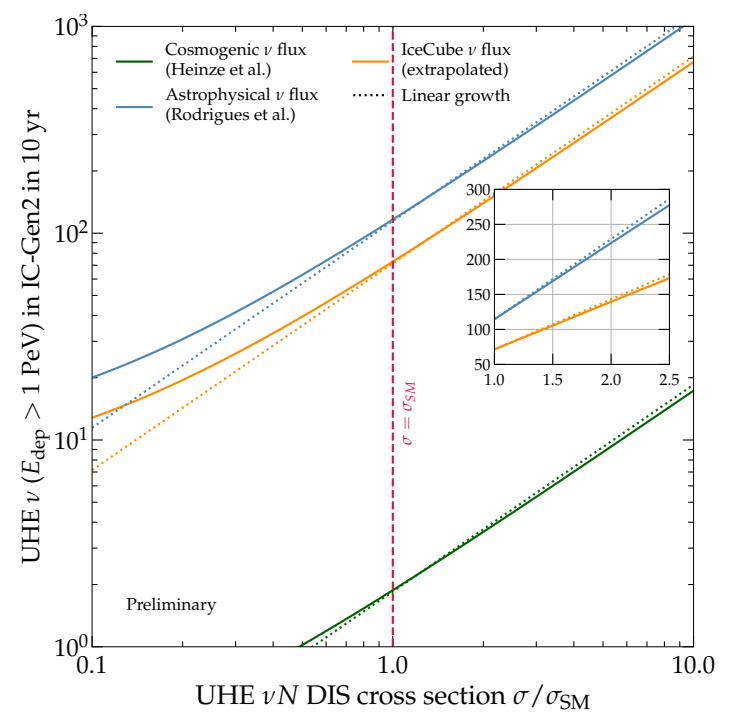

Figure 5: Predicted number of neutrino events with deposited energy $E_{\text {dep }}>1 \mathrm{PeV}$ detected in the radio component of IceCube-Gen2 in 10 years, as a function of the $v N$ cross section for the three benchmark UHE $v$ fluxes from Fig. 1.

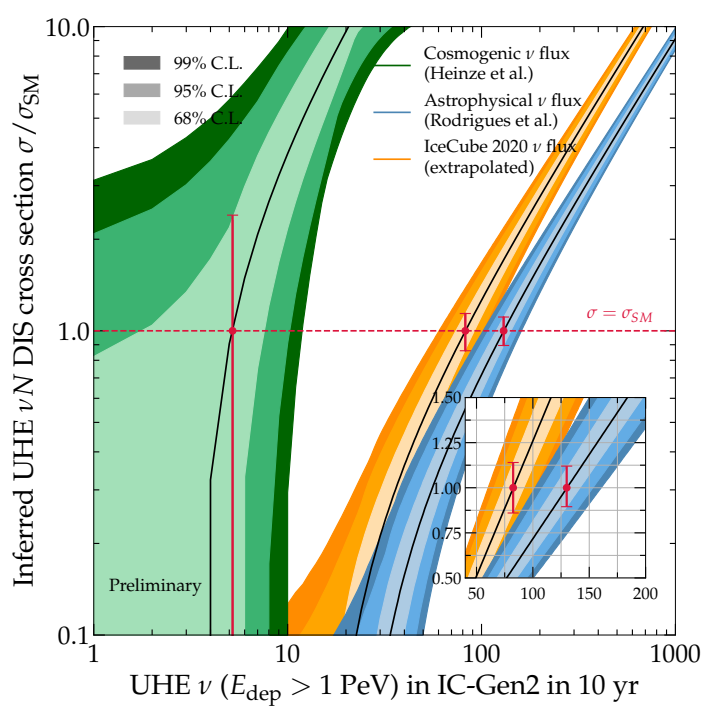

Figure 6: Credible regions of inferred cross section as a function of the number of the number of events observed by IceCube-Gen 2 in 10 years, for the cosmogenic, astrophysical, and IceCube extrapolated fluxes from Fig. 1. Error bars show the $68 \%$ C.L. sensitivity assuming the Standard Model cross section is the true cross section.

\section{Conclusions and outlook}

We have shown the potential of the radio component of IceCube-Gen2 to use UHEv to measure the neutrino-nucleon cross section at $\mathrm{EeV}$ neutrino energies for the first time. We computed the sensitivity to measuring the cross section using a sophisticated end-to-end computation framework, including the most up-to-date UHEv fluxes, attenuation of neutrinos inside the Earth, and estimates of the IceCube-Gen2 performance.

We adopted three benchmark UHEv fluxes: a low cosmogenic flux, a high astrophysical flux, and an intermediate flux, the IC flux extrapolated to high energies. Our results show that IceCubeGen2 will be able to measure the cross section to within $\sim 11 \%$ if the UHE $v$ flux is high. In the future, we will explore possible ways to weaken the dependence of our analysis on the knowledge of the UHE $v$ flux.

\section{References}

[1] IceCube-Gen2 Collaboration, M. G. Aartsen et al. J. Phys. G 48 no. 6, (2021) 060501.

[2] K. Greisen Phys. Rev. Lett. 16 (1966) 748-750.

[3] NuTeV Collaboration, M. Tzanov et al. Phys. Rev. D 74 (2006) 012008.

[4] IceCube Collaboration, M. G. Aartsen et al. Nature 551 (2017) 596-600. 
[5] M. Bustamante and A. Connolly Phys. Rev. Lett. 122 no. 4, (2019) 041101.

[6] IceCube Collaboration, R. Abbasi et al.

[7] P. B. Denton and Y. Kini Phys. Rev. D 102 (2020) 123019.

[8] IceCube Collaboration, C. Haack and C. Wiebusch PoS ICRC2017 (2018) 1005.

[9] J. Heinze, A. Fedynitch, D. Boncioli, and W. Winter Astrophys. J. 873 no. 1, (2019) 88.

[10] X. Rodrigues, J. Heinze, A. Palladino, A. van Vliet, and W. Winter Phys. Rev. Lett. 126 no. 19, (2021) 191101.

[11] A. van Vliet, R. Alves Batista, and J. R. Hörandel Phys. Rev. D 100 no. 2, (2019) 021302.

[12] RNO-G Collaboration, J. A. Aguilar et al. JINST 16 no. 03, (2021) P03025.

[13] K. Fang and K. Murase Nature Phys. 14 no. 4, (2018) 396-398.

[14] V. Bertone, R. Gauld, and J. Rojo JHEP 01 (2019) 217.

[15] M. Ackermann et al. Bull. Am. Astron. Soc. 51 (2019) 215.

[16] R. Gandhi, C. Quigg, M. H. Reno, and I. Sarcevic Astropart. Phys. 5 (1996) 81-110.

[17] A. Cooper-Sarkar and S. Sarkar JHEP 01 (2008) 075.

[18] A. Connolly, R. S. Thorne, and D. Waters Phys. Rev. D 83 (2011) 113009.

[19] C. A. Argüelles, F. Halzen, L. Wille, M. Kroll, and M. H. Reno Phys. Rev. D 92 no. 7, (2015) 074040.

[20] A. Garcia, R. Gauld, A. Heijboer, and J. Rojo JCAP 09 (2020) 025.

[21] D. García-Fernández, A. Nelles, and C. Glaser Phys. Rev. D 102 no. 8, (2020) 083011.

[22] A. Anker et al. JCAP 11 (2019) 030. 